\title{
Efficacy of fluticasone on cough: a randomised controlled trial
}

\author{
B.P. Ponsioen*, W.C.J. Hop ${ }^{\#}$, N.A. Vermue ${ }^{\star}$, P.N.R. Dekhuijzen ${ }^{+}$and A.M. Bohnen*
}

ABSTRACT: Cough may be the consequence of bronchial hyperresponsiveness (BHR) and inflammation. This study was designed to investigate the short-term effects of an inhaled steroid (fluticasone propionate (FP)) on cough, and to determine the effects of smoking, BHR, allergy and forced expiratory volume in one second (FEV 1$)$ on the efficacy of FP.

In a community-based primary healthcare centre, 135 previously healthy adults suffering from cough for $\geqslant 2$ weeks were enrolled in a randomised, double-blind, placebo-controlled trial of inhaled FP $500 \mu \mathrm{g}$ b.i.d. for 2 weeks. Participants completed daily diary cards of lower respiratory tract symptoms. The primary outcome measure was the decrease in mean total daily cough score (0-6) during the second week of treatment.

In the FP group, the cough score decreased from 3.8 at baseline to mean \pm SEM $1.4 \pm 0.2$ during the second week. In the placebo group, this decrease was from 3.8 to $1.9 \pm 0.1$ and was statistically significantly less. A favourable effect of FP was only detectable in nonsmokers, in whom the score was 0.9 points lower compared with placebo. The clinical relevance of this finding has to be established further. Allergy, FEV 1 and BHR at baseline did not affect the efficacy of FP.

In conclusion, anti-inflammatory treatment with the inhaled steroid fluticasone propionate reduces cough in otherwise healthy adults who do not smoke.

KEYWORDS: Adults, cough, fluticasone, primary care, smoking

ough is one of the symptoms of lower respiratory tract (LRT) illness; other symptoms include sputum production, wheeze, shortness of breath and chest tightness [1]. The current authors postulated that inhaled corticosteroids (ICS) will reduce cough in nonasthmatics. Recent findings strongly suggest that the causes and mechanisms of persistence of cough in nonasthmatics may be similar to those in asthmatics [2,3]. First, cough and other LRT symptoms after rhinovirus infections were not only prevalent in asthmatics, but also in nonasthmatics [1]. Secondly, not only asthmatics but also nonatopic nonasthmatic subjects may develop bronchial inflammation after a common cold; a viral infection of the upper respiratory tract [3]. The effects of fluticasone propionate (FP, an anti-inflammatory agent) on cough and other LRT symptoms in asthmatics are seen within 2 weeks [4]. However, cigarette smoking negatively influences this short-term effect of ICS [5]. In smokers with chronic obstructive pulmonary disease (with forced expiratory volume in one second (FEV1): $60 \%$ predicted), a 6-week course of budesonide $1,600 \mu \mathrm{g}$ q.d. did not improve symptoms, lung function or bronchial hyperresponsiveness (BHR) [6].
In the community, the incidence of acute cough, defined as cough lasting $<3$ weeks [7], was 54 out of 1,000 in previously healthy adults [8]. The aim of the current study was to investigate the shortterm effects of inhaled FP $(500 \mu \mathrm{g} \quad$ b.i.d. $)$ versus placebo on cough in previously healthy, nonasthmatic adults with persistence of cough in a community-based primary healthcare centre. The primary outcome measure was the decrease in daily cough score during the 2-week treatment period. Secondary outcomes included the patients' perception of the efficacy of the treatment, the need for subsequent treatment, days off work, nocturnal awakenings, other LRT symptoms, pulmonary function and adverse events. The current authors also aimed to determine the effects of smoking, BHR, allergy and FEV1 on efficacy of FP in order to elucidate its mechanism of action.

\section{SUBJECTS AND METHODS \\ Patients}

The study was a 2-week, double-blind, parallelgroup, randomised trial in a primary healthcare centre (six practices; Brielle, The Netherlands) with 10,262 patients aged 18-65 yrs (table 1). Inclusion criteria were as follows: aged 18-65 yrs, cough of

\section{AFFILIATIONS}

Depts of *General Practice, and \#Epidemiology and Biostatistics, ErasmusMC, University Medical Center Rotterdam, Rotterdam, and -GlaxoSmithKline, Zeist, and +Dept of Pulmonary Diseases, University Medical Center, Nijmegen, The Netherlands.

CORRESPONDENCE

B.P. Ponsioen,

Dept of General Practice of ErasmusMC

University Medical Center Rotterdam Room FF 323, P0 Box 1730

3000 DR Rotterdam

The Netherlands

Fax: 31181413116

E-mail: b.ponsioen@erasmusmc.nl

Received:

May 052004

Accepted after revision:

August 212004

\section{SUPPORT STATEMENT}

This study was supported by GlaxoSmithKline. GlaxoSmithKline were involved in the data collection, but had no role in the analysis/ interpretation of data. 


\begin{tabular}{|c|c|c|c|}
\hline \multirow[t]{2}{*}{ TABLE 1} & \multicolumn{3}{|c|}{$\begin{array}{l}\text { Characteristics of } 133 \text { analysed patients receiving } \\
\text { placebo or fluticasone }\end{array}$} \\
\hline & & Placebo & Fluticasone \\
\hline \multicolumn{2}{|l|}{ Subjects $n$} & 68 & 65 \\
\hline \multicolumn{2}{|l|}{ Female $n$} & 45 & 41 \\
\hline \multicolumn{2}{|l|}{ Age yrs } & $43.4(18-65)$ & $47.0(19-64)$ \\
\hline \multicolumn{4}{|l|}{ Cough } \\
\hline \multicolumn{2}{|c|}{ Baseline cough score (scale: $0-6$ ) } & $3.8 \pm 1.0$ & $3.8 \pm 1.0$ \\
\hline \multicolumn{4}{|c|}{ Duration } \\
\hline \multicolumn{2}{|c|}{$<3$ weeks (acute) } & $18 / 68$ & $13 / 65$ \\
\hline \multicolumn{2}{|c|}{ 3-8 weeks (subacute) } & $41 / 68$ & $48 / 65$ \\
\hline \multicolumn{2}{|c|}{ 8-17 weeks (chronic) } & $9 / 68$ & $4 / 65$ \\
\hline \multicolumn{4}{|c|}{ Other daytime LRT symptoms } \\
\hline \multicolumn{2}{|c|}{ Sputum production } & $49 / 65$ & $46 / 65$ \\
\hline \multicolumn{2}{|l|}{ Wheeze } & $19 / 64$ & $24 / 65$ \\
\hline \multicolumn{2}{|c|}{ Shortness of breath } & $38 / 63$ & $32 / 65$ \\
\hline \multicolumn{2}{|c|}{ Tightness of the chest } & $42 / 63$ & $41 / 65$ \\
\hline \multicolumn{2}{|c|}{ Hoarseness mild/moderate } & $10 / 68$ & $12 / 65$ \\
\hline \multicolumn{2}{|c|}{ Current smoker } & $24 / 67(36)$ & $24 / 65$ (37) \\
\hline \multicolumn{2}{|c|}{ Rhonchi (scattered) \# } & $9 / 68$ & $10 / 65$ \\
\hline \multicolumn{4}{|c|}{ Lung function } \\
\hline \multicolumn{2}{|c|}{$\mathrm{FEV}_{1} \%$ pred } & $102 \pm 18$ & $102 \pm 14$ \\
\hline \multicolumn{2}{|c|}{$\mathrm{FEV}_{1} / \mathrm{FVC} \%$} & $81 \pm 8$ & $82 \pm 6$ \\
\hline \multicolumn{2}{|c|}{$\mathrm{PD}_{20}<\mathbf{2} \mathbf{m g}^{+}$} & $26 / 65(40)$ & $18 / 64(28)$ \\
\hline \multicolumn{4}{|l|}{ Allergy } \\
\hline \multicolumn{2}{|c|}{ Phadiatop positive } & $15 / 68$ & $16 / 65$ \\
\hline \multicolumn{2}{|c|}{ History of allergy of airways } & $16 / 65(25)$ & $13 / 65(20)$ \\
\hline \multicolumn{4}{|l|}{ Infection } \\
\hline \multicolumn{2}{|c|}{ Serological evidence of infection } & $56 / 68$ & $52 / 64$ \\
\hline \multicolumn{2}{|c|}{$\mathrm{CRP}>10 \mathrm{mg} \cdot \mathrm{L}^{-1}$} & $14 / 66$ & $11 / 64$ \\
\hline \multicolumn{2}{|c|}{$\mathrm{CRP}>50 \mathrm{mg} \cdot \mathrm{L}^{-1}$} & $0 / 66$ & $2 / 64$ \\
\hline \multicolumn{2}{|c|}{ Self-reported preceding common cold } & $42 / 59^{\S}$ & $46 / 59^{\S}$ \\
\hline
\end{tabular}

Data are presented as $n$, mean (range), mean $\pm S D, n / n$ and $n / n$ (\%). LRT: lower respiratory tract; $F E V_{1}$ : forced expiratory volume in one second; FVC: forced vital capacity; PD20: dose of histamine provoking a fall in FEV 1 of $\geqslant 20 \%$; CRP: C-reactive protein. ${ }^{\#}$ : auscultation of the chest; " : using European reference values [9]; ${ }^{+}$: a measure of bronchial hyperresponsiveness; ${ }^{\text {s. }}$ : first 15 patients not assessed.

$>2$ weeks duration, and informed consent. Patients completed a daily diary card for cough (score $0=$ absent, $1=$ mild, $2=$ moderate, 3 =severe) [10-12] and other LRT symptoms regarding the previous day and night. Only patients with a night score of $\geqslant 1$ point and a combined day plus night score of $\geqslant 3$ points were included. Exclusion criteria were as follows: 1) history of asthma; 2) incidences of self-reported wheeze, pharmacy data indicating asthma-like symptoms or variability in lung function in the previous year [13]; 3) current treatment that might influence the cough; 4) $\mathrm{FEV} 1<60 \%$ predicted; 5) any concurrent airway disease (e.g. pneumonia, cancer, tuberculosis, tonsillitis, sinusitis); 6) uncontrolled systemic disease or pregnancy; and 7) patients previously randomised for the study. The protocol was approved by the ethics committee of the Erasmus Medical Centre, Rotterdam, The Netherlands. Written informed consent was obtained from all patients.

\section{Methods}

When patients were referred from the participating physicians for the study, the investigator (B.P. Ponsioen) documented baseline data, medical history, concurrent medication and results of a physical examination. On the same day, FEV1, forced vital capacity (FVC) and BHR were measured by pneumotachography (Jaeger Masterscreen System; Jaeger, Wurzburg, Germany). BHR was assessed by measuring the dose of histamine aerosol that lowers FEV1 by $20 \%$ from the patients' baseline value (PD20) [14]. Presence of BHR was defined as PD20 $<2.0 \mathrm{mg}$ histamine. A blood sample was taken and sent to the laboratory to test for C-reactive protein and Phadiatop (seroallergy).

Blinded study medication was provided by GlaxoSmithKline (Evreux, France) according to a computer-generated randomisation list. Either two puffs of $250-\mu \mathrm{g}$ metered-dose inhaler (MDI) of FP or placebo MDI to match FP were inhaled via Volumatic spacer (Betts Company, Colchester, UK) in the morning and the evening for 2 weeks. This dose and application was chosen to investigate whether or not antiinflammatory treatment is efficacious without too high a risk for adverse reactions. No rescue medication was allowed. Compliance was checked by weighing the canisters before and after the treatment period, and the patients were asked whether they had any problems using the study medication. The patients were instructed to wash the spacer, if necessary, with warm water and to dry it in room air. Patients were shown how to complete the daily record card (DRC) for the symptoms: cough, sputum production, wheezing, shortness of breath and chest tightness; the scores were $0=$ no symptoms ranging to $3=$ severe symptoms. Number of awakenings at night, days off work and the numbers of cigarettes smoked were also recorded in the DRC. This was to be completed every morning and every evening during the 2 weeks of treatment. Any concurrent medication for LRT symptoms was to be registered in the diary.

After the 2-week treatment period, the patient was asked whether the coughing problems had strongly improved, improved, not changed or increased. Measurements of FEV1, FVC and BHR were repeated. Patients who requested subsequent treatment for cough were supplied with open-label FP $2 \times 250 \mu \mathrm{g} \cdot$ puff $^{-1}$ MDI b.i.d. for the next 2 weeks.

\section{Statistical analysis}

According to the statistical analysis plan, the primary endpoint was the mean daily cough score during the second week of treatment. The daily cough score equals the sum of the separate daytime and night-time scores and ranges $0-6$. The primary comparison of the two groups was planned using analysis of covariance (ANCOVA) with the baseline cough score, smoking status (yes/no) and BHR (yes/no) as covariates. Effect modification by these factors was evaluated using appropriate interaction terms. According to the protocol, additional subgroup analyses were planned for smoking status and BHR. The intention-to-treat analysis comprised all randomised patients except those without any follow-up data $(n=2)$. Since the second visit to the centre was planned after 2 weeks ( \pm 3 days), some patients did not complete the full 2week period in the diary. For these cases, the last-observationcarried-forward approach was used to complete the full 
2-week period. However, the conclusions from this analysis were not sensitive to these extrapolations because an alternative analysis of the last 7 days of treatment gave results that negligibly differed from the results presented. In the per protocol analysis, only those patients who had completed the treatment period without major protocol violations were evaluated. The severity of protocol violations was assessed by the investigator before the code was broken.

Secondary endpoints were as follows: the percentage of patients in which the mean cough score during the second week was reduced by $>50 \%$ compared with baseline cough score; the patients' perception of the efficacy of the treatment (compared with the Mann-Whitney U-test); the requirement for additional medication after the treatment period (Fisher's exact test); and days off work (Mann-Whitney U-test). Comparisons between treatment and placebo group of the diary cough scores on each treatment day were also performed with the Mann-Whitney U-test. Further exploratory analyses were carried out on the effects on the diary sputum production scores, wheezing scores, chest tightness scores, BHR and FEV1. A p-value of 0.05 (two-sided) was considered statistically significant. Power calculations indicated that 120 patients were required, but, to allow for dropouts, the current authors decided to enrol 135 patients.

\section{Role of the funding source}

The study sponsor, GlaxoSmithKline, monitored data collection in the case record forms written by the investigator during the trial period and transformed the data in an electronic database afterwards. GlaxoSmithKline had no role in the analysis and interpretation of the data, neither did the study sponsor have a role in the writing of the report and in the decision to submit the paper for publication.

\section{RESULTS}

\section{Baseline characteristics}

The recruitment period was from February 212000 to June 21 2001 (fig. 1). Of the possible 418 patients, 133 were not recruited because of a lack of night symptoms or a current need for antibiotics. Most of those who declined to participate (74 out of 418) were patients who did not want to miss active treatment for another 2 weeks. Of the 135 randomised patients, 133 were evaluable (no follow-up data were available for two patients). The second visit took place after $\geqslant 11$ days of treatment in 131 of the 133 patients. Baseline data on the 133 evaluated patients are given in table 1 . No clinically relevant differences were found between the two study groups.

\section{Primary analysis}

Intention-to-treat analysis

In the FP group, the mean \pm SEM cough score decreased from 3.8 at baseline to $1.4 \pm 0.2$ during the second week of treatment. The corresponding figures in the placebo group were 3.8 and $1.9 \pm 0.1$, respectively. Overall, the difference between the two groups was significant $(p=0.012)$. Adjusted for BHR, smoking status and baseline cough score, ANCOVA showed that the average daily cough score in the second week of treatment was 0.5 points (95\% confidence interval (CI): $0.1-0.9$ ) lower in patients using FP than in the placebo group $(p=0.019)$.

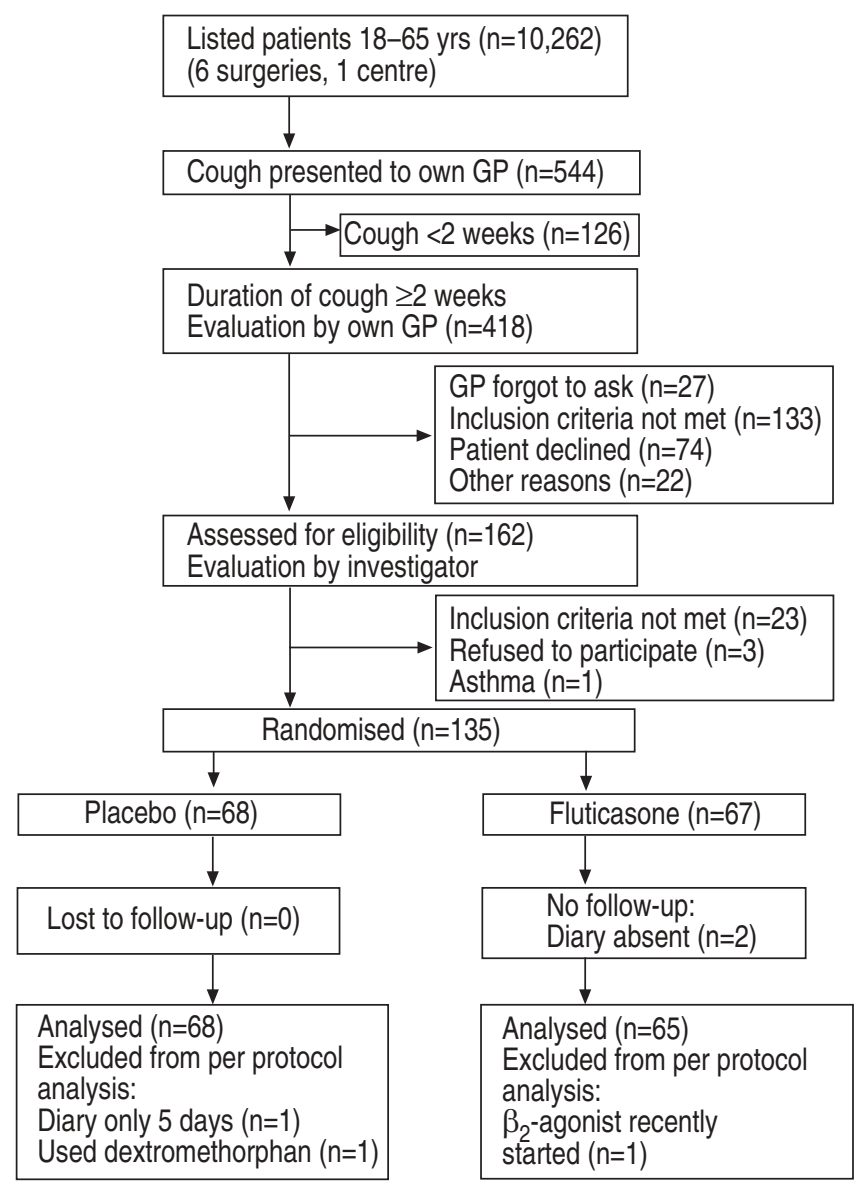

FIGURE 1. Trial profile. GP: general practitioner.

However, further analysis showed that the difference between FP and placebo was significantly $(p=0.015)$ related to smoking status and not to BHR or baseline cough score. Separate analysis of nonsmokers (64\% of patients) and smokers showed that, adjusted for baseline cough score, for nonsmoking patients using FP, the average daily cough score in the second week was 0.9 points (95\% CI: $0.4-1.3$ ) lower than in the placebo group $(\mathrm{p}<0.001)$. This treatment effect was not significantly related to the baseline cough score. Considering the entire treatment period, the difference between both study groups was significant from day 5 onwards in nonsmokers (fig. 2). In smokers, patients using FP had an average cough score during the second week that was 0.1 point higher as compared with placebo ( $p=0.74 ; 95 \% \mathrm{CI}$ : $-0.6-0.9$; fig. 2 ). The effect of FP on the reduction in cough score was not significantly influenced by BHR; this applied both to smokers and nonsmokers.

Further analysis using ANCOVA showed that the presence of allergy (positive Phadiatop), rhonchi on auscultation, FEV1 $<100 \%$ predicted, and a duration of cough of $\leqslant 4$ weeks (the median of cough duration at baseline) did not significantly affect the difference between the two treatment groups. Again, this applied to smokers and nonsmokers.

Per protocol analysis

In the per protocol analysis, three patients were excluded (fig. 1); one patient left the study after 5 days due to emotional 

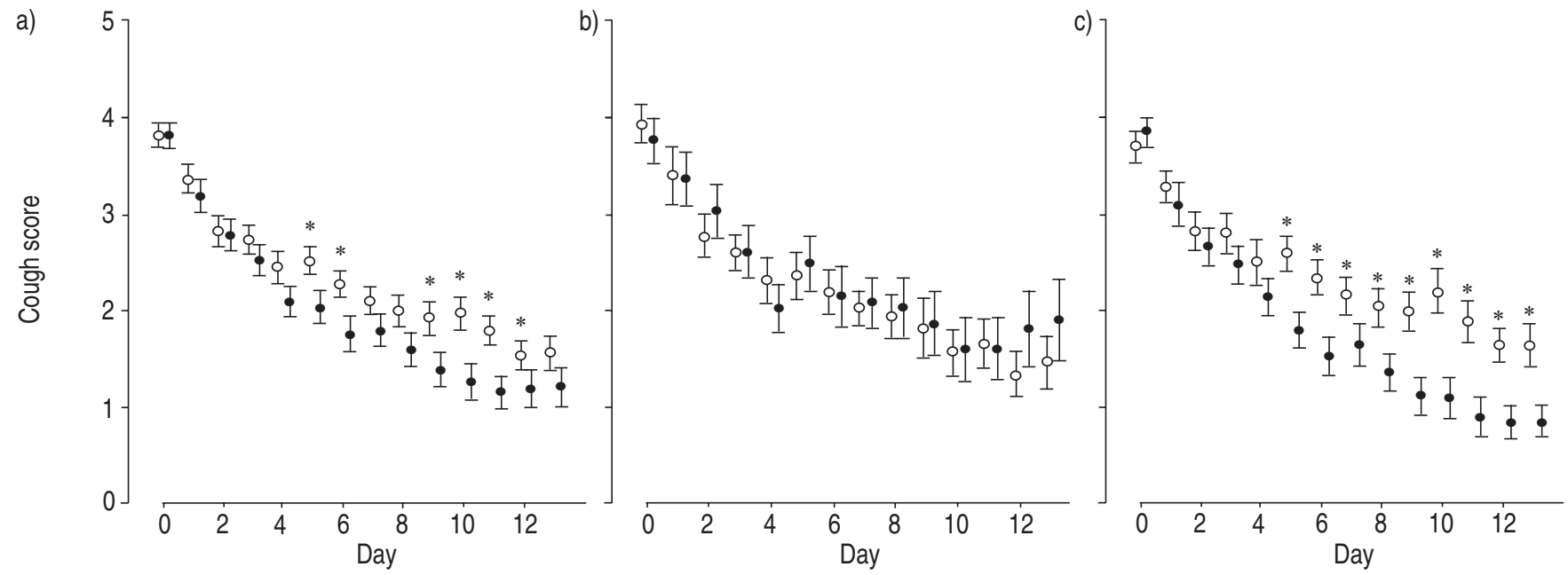

FIGURE 2. Mean \pm SEM of daily cough score (scale 0-6) during the 2-week treatment period. Scores are given for a) the total group, and the subgroups of b) smokers and c) nonsmokers. Day 0 corresponds with baseline cough score.

: fluticasone; $\bigcirc$ : placebo. *: $p<0.05$ placebo compared with fluticasone.

problems, one patient used dextromethorphan during the study, and the third patient was found to have started a $\beta_{2}$-antagonist shortly before entry into the study. Re-analysis of the data gave similar results as presented in the intention-totreat analysis.

Medication compliance was estimated from the difference in weight of the canisters before and after the treatment. Of the patients in the FP group, 58 out of 65 had used $>80 \%$ of the recommended dose, compared with 63 out of 68 in the placebo group. In the FP group, the mean daily inhaled FP dose showed no significant correlation with the decrease in cough score.

\section{Secondary analysis}

The proportion of nonsmokers in which the mean cough score in the second week was reduced by $>50 \%$ was 23 out of 43 $(54 \%)$ in the placebo group and 33 out of 41 (81\%) in the FP group $(\mathrm{p}=0.011)$. This resulted in a number needed to treat of 3.7. The perception of the efficacy of the treatment was significantly better in the FP group compared with the placebo group $(p=0.001)$. In nonsmokers, the perception of improvement by FP was greater $(p=0.004)$ than in the smokers $(\mathrm{p}=0.56)$. Additional treatment after 2 weeks of study medication was requested in $43 \%$ ( 28 out of 65 ) of the patients in the FP group and in $63 \%$ (42 out of 67 ) of the patients in the placebo group $(\mathrm{p}=0.036)$. These percentages for nonsmokers were $39 \%$ and $68 \%$, respectively $(p=0.014)$, compared with $50 \%$ and $54 \%$ for smokers, respectively. No significant differences between placebo and FP (smokers and nonsmokers) were found for the effects on days off work, nocturnal awakenings, LRT symptoms (except cough and sputum), rhonchi, FEV1 and PD20. The average daytime sputum score $(0-3)$ in the second week of treatment was 0.3 points lower in patients using FP than in the placebo group $(p<0.05)$. Subgrouped according to smoking status, it was found that there was no significant difference in smokers, whilst the difference in the means of the sputum score was 0.4 in nonsmokers $(\mathrm{p}<0.01)$.

\section{Side-effects}

At baseline, the frequency of hoarseness was similar in both groups (table 1). There were no differences in the frequency of hoarseness during the treatment period (10 out of 68 and 9 out of 65 after the treatment with placebo and FP, respectively). There were no significant differences between the two groups in the incidence of adverse events that were considered to be related to the study medication. In the FP group, oropharyngeal candidiasis was found in one patient, and two patients reported hoarseness as an adverse event. In the placebo group, one patient reported hoarseness and another patient reported sore throat as an adverse event. Neither of the groups had any serious adverse events that were related to the treatment.

\section{DISCUSSION}

The trial shows that FP improves cough scores from day 5 onwards in previously healthy nonsmoking adults who consulted their general practitioner (GP) with cough for $\geqslant 2$ weeks. Most of the patients included had subacute cough at baseline. Allergy, FEV1 and BHR at baseline did not affect the efficacy of FP.

MACFARLANE et al. [8] studied adults who presented with cough that had been present $\leqslant 21$ days and evidence of infection $(50 \%$ of the cases). Neither "infection" nor antibiotic prescription was related to the outcome of the cough. The benefits of antibiotics in cough do not outweigh their side-effects [15]. The effectiveness of the centrally acting drugs, codeine and dextromethorphan, has not yet been fully elucidated [16]. The effects of $\beta_{2}$-agonists have only been detected in small subgroups of patients with evidence of airflow obstruction (abnormalities on auscultation, FEV $1<80 \%$ predicted) or $\mathrm{BHR}$ $[11,16,17]$

\section{Methodological issues}

Objective markers of cough (e.g. capsaicin test) [18] are not available in the primary care setting, and cough-specific quality of life measurements are under development $[19,20]$. An extremely large placebo effect of antitussive drugs was shown over the first 2-5 days of treatment of acute cough [21]. 


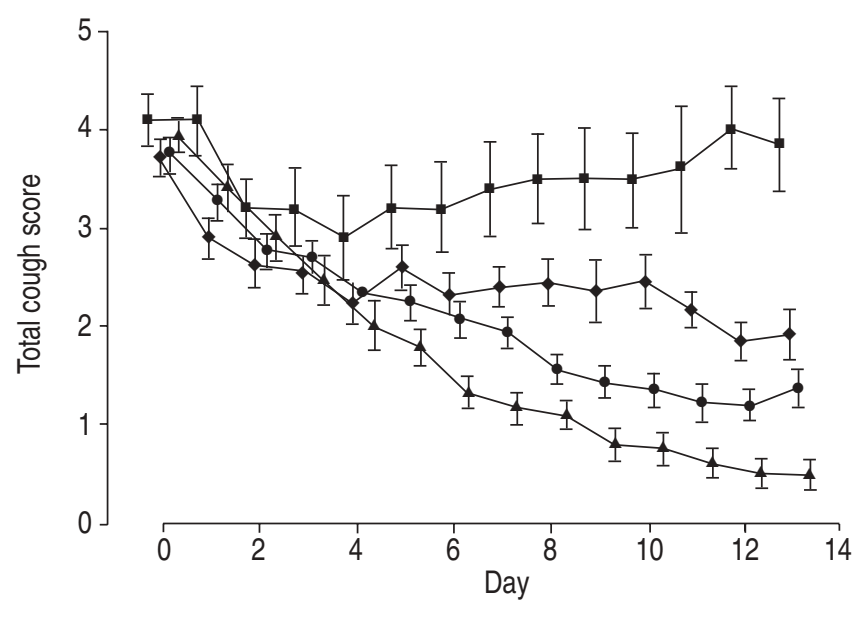

FIGURE 3. Relationship between the cough score (0-6) recorded on the daily record card and patients' global evaluation of improvement of cough symptoms after the treatment. : worse cough symptoms; : no improvement of symptoms; - : some improvement of symptoms; $\mathbf{\Lambda}$ : much improvement of symptoms.

The primary outcome measure, the daily cough score, is a subjective one.

However, cough scores have been validated against microphone recorders and digital-sound pressure level meters for cough frequency and intensity [21]. Moreover, there was a good correlation between the daily cough score in the DRC and the patients' perception of efficacy after the 2-week treatment period (fig. 3).

A minority of patients with unclear respiratory symptoms might have asthma [22]. However, the results of the current study in patients with cough show that patients with asthma were successfully excluded for the following reasons. 1) Neither BHR nor seroallergy predicted improvement in cough scores by FP. 2) There was no effect of FP on BHR in the current patients after 2 weeks of treatment with FP, whereas FP in mild persistent asthmatics achieves a reduction in BHR within 3 days of starting treatment [23]. 3) The values of FEV1 and the frequency of seroallergy among the participants of the current study correspond well with data from population surveys [24]. 4) The incidence of persistent cough in the current study is consistent with the results of other primary care studies $[8,25] .5)$ The prevalence of BHR was $34 \%$ at baseline and was reduced to $18 \%$ after 2 weeks. The latter may reflect the prevalence of BHR in the Dutch adult population in which BHR (defined as PD20 $\leqslant 1 \mathrm{mg}$ metacholine) is prevalent in 11$14 \%$ [24].

\section{Pathophysiological mechanisms related to effects of fluticasone propionate}

Interpretation of the mechanism of action of FP in the current patients is difficult, as cough-reflex testing and direct assessment of airway inflammation were missing in this study. Asthma research shows that "the relation between airway responsiveness and airway inflammation is complex, depending on acute and chronic inflammatory events as well as intrinsic smooth muscle factors" [26]. Cough and hypersecretion may result from inflammation localised in the epithelium and lamina propria of the airways [27]. In patients with a range of severity of lung disease caused by cigarette smoking, ICS are largely ineffective in reducing neutrophil-mediated inflammation [28]. Perhaps the nonsmokers in the current study responded better to FP because nonsmokers may have a heightened baseline cough-reflex sensitivity compared with that of smokers, as suggested by DicPINIGAITIS [29]. The finding that a drug with well-studied anti-inflammatory action, like $\mathrm{FP}$, reduces sputum production in nonsmokers with cough suggests a short-term, topical anti-inflammatory effect of FP in the studied patients.

Severity of baseline cough score was not related to the treatment effect, indicating that FP has a constant effectiveness in mild, moderate and severe cough. Since there was no doseeffect relationship, it was assumed that FP at a dose of $500 \mu \mathrm{g}$ b.i.d. was sufficient. The small noncompliance that was registered did not negatively influence the effect of FP. In asthma, the recommended dose of FP depends on severity (100-500 $\mu$ g b.i.d.).

Further studies into the action of FP in cough, including inflammatory markers and cough-reflex sensitivity testing, are needed to support the relevance of inflammation in cough.

\section{Clinical relevance of the effects of fluticasone propionate in persistent cough}

The benefits from inhaled FP in nonasthmatic nonsmokers with cough seem to be modest. The proportion of patients who requested additional medication after the 2-week treatment period was 28 out of 65 in patients who had used FP compared with 42 out of 67 in patients who had used placebo.

During the additional treatment with FP, there were no differences in the proportion of subjects with resolution of cough between the group that had used FP and the group that had used placebo during the first 2 weeks.

Persistent cough, as diagnosed by the GP, is a self-limiting disease. Therefore, the cost of prescribed drugs should carefully be weighted against the level of inconvenience of the symptoms and the expected effect of the prescribed drug. Most patients with persistent cough re-consult their GP because they feel bothered by their LRT symptoms [30]. A difference in the current primary outcome measure, the cough score during the second week, of 0.9 points lower in nonsmokers using FP compared with nonsmokers using placebo could be clinically relevant. The results of the present trial stress the need for further study of the clinical relevance and the cost-benefit ratio of FP in nonsmoking patients who present with bothersome cough.

In conclusion, this study shows that the anti-inflammatory agent fluticasone propionate reduces cough in nonsmoking previously healthy adults. This trial supports the view that smoking attenuates the effect of inhaled corticosteroids on cough. The cost-benefit ratio of fluticasone propionate in nonsmoking patients who present with bothersome cough needs further study.

\section{ACKNOWLEDGEMENTS}

The authors would like to thank all of the patients who participated in this trial, and all of the nurses, GPs (J.J. van 
Binsbergen, G.J. van den Heuvel, A.J.P.M. van den Hurk, G.M. Schoenmaker, C.J. in t'Veld, H.K. Winkel) and chemists (R. Ardon, R.H. Rouws) of the primary healthcare centre (Huisartsengroep Brielle, Brielle, The Netherlands). R. vd Polder, practice nurse, managed the patients' visits and performed the lung function measurements.

\section{REFERENCES}

1 Corne JM, Marshall C, Smith S, et al. Frequency, severity, and duration of rhinovirus infections in asthmatic and non-asthmatic individuals: a longitudinal cohort study. Lancet 2002; 359: 831-834.

2 Gibson PG, Fujimura M, Niimi A. Eosinophilic bronchitis: clinical manifestations and implications for treatment. Thorax 2002; 57: 178-182.

3 Trigg CJ, Nicholson KG, Wang JH, et al. Bronchial inflammation and the common cold: a comparison of atopic and non-atopic individuals. Clin Exp Allergy 1996; 26: 665-676.

4 Szefler SJ, Boushey HA, Pearlman DS, et al. Time to onset of effect of fluticasone propionate in patients with asthma. J Allergy Clin Immunol 1999; 103: 780-788.

5 Chalmers GW, Macleod KJ, Little SA, Thomson LJ, McSharry CP, Thomson NC. Influence of cigarette smoking on inhaled corticosteroid treatment in mild asthma. Thorax 2002; 57: 226-230.

6 Rutgers SR, Koeter GH, van der Mark TW, Postma DS. Short-term treatment with budesonide does not improve hyperresponsiveness to adenosine $5^{\prime}$-monophosphate in COPD. Am J Respir Crit Care Med 1998; 157: 880-886.

7 Irwin RS, Madison JM. The diagnosis and treatment of cough. N Engl J Med 2000; 343: 1715-1721.

8 Macfarlane J, Holmes W, Gard P, et al. Prospective study of the incidence, aetiology and outcome of adult lower respiratory tract illness in the community. Thorax 2001; 56: 109-114.

9 Quanjer PH, Tammeling GJ, Cotes JE, Pedersen OF, Peslin R, Yernault JC. Lung volumes and forced ventilatory flows. Report Working Party Standardization of Lung Function Tests, European Community for Steel and Coal. Official Statement of the European Respiratory Society. Eur Respir J 1993; 6: Suppl. 16, 5-40.

10 Lee PCL, Jawad MS, Eccles R. Antitussive efficacy of dextromethorphan in cough associated with acute upper respiratory tract infection. J Pharm Pharmacol 2000; 52: 1137-1142.

11 Tukiainen $H$, Karttunen $P$, Silvasti $M$, et al. The treatment of acute transient cough: a placebo-controlled comparison of dextromethorphan and dextromethorphan-beta 2-sympathomimetic combination. Eur J Respir Dis 1986; 69: 95-99.

12 Berkowitz RB, Connell JT, Dietz AJ, Greenstein SM, Tinkelman DG. The effectiveness of the nonsedating antihistamine loratadine plus pseudoephedrine in the symptomatic management of the common cold. Ann Allergy 1989; 63: 336-339.

13 Tattersfield AE, Knox AJ, Britton JR, Hall IP. Asthma. Lancet 2002; 360: 1313-1322.

14 Sterk PJ, Fabbri LM, Quanjer PH, et al. Airway responsiveness. Standardized challenge testing with pharmacological, physical and sensitising stimuli in adults. Report
Working Party Standardization of Lung Function Tests, European Community for Steel and Coal. Official Statement of the European Respiratory Society. Eur Respir J Suppl 1993; 16: 53-83.

15 Evans AT, Husain S, Durairaj L, Sadowski LS, CharlesDamte M, Wang Y. Azithromycin for acute bronchitis: a randomised, double-blind, controlled trial. Lancet 2002; 359: 1648-1654.

16 Schroeder K, Fahey T. Systematic review of randomised controlled trials of over the counter cough medicines for acute cough in adults. BMJ 2002; 324: 329-331.

17 Smucny J, Fahey T, Becker L, Glazier R, McIsaac W. Antibiotics for acute bronchitis. Cochrane Database Syst Rev 2004; 4: CD000245.

18 Di Franco A, Dente FL, Giannini D, et al. Effects of inhaled corticosteroids on cough threshold in patients with bronchial asthma. Pulm Pharmacol Ther 2001; 14: 35-40.

19 French CT, Irwin RS, Fletcher KE, Adams TM. Evaluation of a cough-specific quality-of-life questionnaire. Chest 2002; 121: 1123-1131.

20 Birring SS, Prudon B, Carr AJ, Singh SJ, Morgan MD, Pavord ID. Development of a symptom specific health status measure for patients with chronic cough: Leicester Cough Questionnaire (LCQ). Thorax 2003; 58: 339-343.

21 Freestone C, Eccles R. Assessment of the antitussive efficacy of codeine in cough associated with common cold. J Pharm Pharmacol 1997; 49: 1045-1049.

22 Ternesten-Hasseus E, Farbrot A, Lowhagen O, Millqvist E. Sensitivity to methacholine and capsaicin in patients with unclear respiratory symptoms. Allergy 2002; 57: 501-507.

23 Sovijarvi AR, Haahtela T, Ekroos HJ, et al. Sustained reduction in bronchial hyperresponsiveness with inhaled fluticasone propionate within three days in mild asthma: time course after onset and cessation of treatment. Thorax 2003; 58: 500-504.

24 Chinn S, Burney P, Jarvis D, Luczynska C. Variation in bronchial responsiveness in the European Community Respiratory Health Survey (ECRHS). Eur Respir J 1997; 10: 2495-2501.

25 Thiadens HA, de Bock GH, Dekker FW, et al. Identifying asthma and chronic obstructive pulmonary disease in patients with persistent cough presenting to general practitioners: descriptive study. BMJ 1998; 316: 12861290.

26 Green RH, Brightling CE, Wardlaw AJ, Pavord ID. Asthma exacerbations and sputum eosinophil counts. Lancet 2003; 361: 1303.

27 Wardlaw AJ, Brightling CE, Green R, Woltmann G, Bradding P, Pavord ID. New insights into the relationship between airway inflammation and asthma. Clin Sci (Lond) 2002; 103: 201-211.

28 Cox G, Whitehead L, Dolovich M, Jordana M, Gauldie J, Newhouse MT. A randomized controlled trial on the effect of inhaled corticosteroids on airways inflammation in adult cigarette smokers. Chest 1999; 115: 1271-1277.

29 Dicpinigaitis PV. Cough reflex sensitivity in cigarette smokers. Chest 2003; 123: 685-688.

30 Holmes WF, Macfarlane JT, Macfarlane RM, Hubbard R. Symptoms, signs, and prescribing for acute lower respiratory tract illness. Br J Gen Pract 2001; 51: 177-181. 